\title{
Lapurdum
}

LAPURDUM Euskal ikerketen aldizkaria | Revue d'études basques |

Revista de estudios vascos | Basque studies review

$7 \mid 2002$

Numéro VII

\section{Articles en basque parus en 1843, dans Le Patriote Français de Montevideo}

\section{Claude Méhats}

\section{OpenEdition \\ Journals}

Édition électronique

URL : http://journals.openedition.org/lapurdum/1011

DOI : 10.4000/lapurdum. 1011

ISSN : 1965-0655

Éditeur

IKER

Édition imprimée

Date de publication : 1 octobre 2002

Pagination : 247-257

ISBN : 2-86781-321-2

ISSN : $1273-3830$

Référence électronique

Claude Méhats, «Articles en basque parus en 1843, dans Le Patriote Français de Montevideo », Lapurdum [En ligne], 7 | 2002, mis en ligne le 01 juin 2009, consulté le 19 avril 2019. URL : http:// journals.openedition.org/lapurdum/1011; DOI : 10.4000/lapurdum.1011 


\section{Articles en basque parus en 1843, dans Le Patriote Français de Montevideo}

Claude MEHATS

Doctorant Université Michel de Montaigne Bordeaux 3

Les quatre textes en langue basque présentés dans cet article sont issus du Patriote Français, un journal imprimé à Montevideo en Uruguay durant la guerre civile communément appelée Guerra grande. Afin de bien cerner le climat politique régnant dans la zone de la Plata à cette époque, nous allons effectuer une présentation des principaux belligérants, suivie par une explication des positionnements stratégiques français. Les causes de la publication de ces textes seront alors plus claires.

L'Uruguay a acquis son indépendance après le soulèvement dit des " 33 orientaux" en 1825. Cette rébellion a pris fin le 20 février 1827 grâce à la victoire décisive d'Ituzaingo face aux Brésiliens. L'année suivante le traité de Rio de Janeiro (27 août 1828 ) est venu confirmer ce nouveau pays qui durant tout le $\mathrm{XIX}^{\text {èmc }}$ siècle va être troublé par une instabilité chronique et par de nombreuses guerres civiles. Vers 1838 se formèrent deux partis : les colorados (libéraux, surnommés colorés à cause de leur insigne, alors que leurs adversaires en portaient un blanc) de Rivera et les blancos (conservateurs) d'Oribe.

Militaire tour à tour luttant pour l'Uruguay (1811), pour le Brésil, à nouveaù pour l'armée uruguayenne lors de l'insurrection des 33 orientaux, José Fructuoso Rivera abandonna celle-ci en 1828 après un désaccord avec Lavalleja. A Buenos Aires, il choqua par ses idées fédérales, avant de devenir gouverneur de la province de Misiones. Président de la république d'Uruguay à sa naissance (1830), il employa son mandat à lutter contre les fédéralistes et contre les Indiens Charruas (qui furent pratiquement exterminés.)

En 1834, lui succéda Manuel Cerefino Oribe, son ancien ministre de la guerre. Ce dernier était également l'un des fameux "33 orientaux" de 1825. Président, il tenta d'étendre le pouvoir du gouvernement sur les immenses possessions de Rivera et l'accusa de fraudes financières durant son mandat. Rivera se rebella, et à la tête du parti coloré, il obtint la victoire de Palmar, contraignant son rival à démissionner en 1838, récupérant par la même occasion la présidence de la république. S'étant retiré à Buenos Aires, Oribe dénonça la nullité de sa renonciation et obtint l'appui armé du dictateur argentin Rosas, qui l'utilisait pour traquer et réprimer ses opposants qui étaient réfugiés en Uruguay. 
Rivera s'unit à la province de Corrientes et à d'autres provinces riveraines et déclara la guerre à Rosas. Après une campagne militaire catastrophique, conclue par la défaite d'Arroyo Grande en 1842, le parti des colorés se retrouva assiégé dans la ville de Montevideo (fortifiée en 60 jours par le général Paz) au début de l'année 1843. Le $1^{\text {er }}$ mars 1843 , Joaquin Suarez en tant que président du sénat remplaça le général Rivera dans l'exercice du pouvoir exécutif et mit en place le gouvernement de la défense (Cooligan Maria Luisa, Arteaga Juan José, 1992). L'amiral argentin Brown entama un blocus maritime mais le commodore anglais Purvis immobilisa les navires argentins. Rosas protesta auprès de Mandeville en signifiant que la confédération était en bonnes relations avec l'Angleterre, mesure qui entraîna Purvis à se retirer. Sur terre, Oribe prit place pour un siège qui devait léser les intérêts commerciaux européens.

En France, la politique extérieure du roi Louis-Philippe qui avait déjà contraint Adolphe Thiers à remettre sa démission par deux fois pour avoir soutenu une politique extérieure jugée trop aventureuse et pouvant mener à des conflits, devint donc timorée sous la direction de Guizot, qui, pour combler son souverain, maintint la paix au prix de concessions et d'humiliations, en particulier vis-à-vis de l'Angleterre. En Uruguay, le relais de cette politique a été assuré par le consul du roi, Théodore Pichon. Au début du siège, il convoque ses concitoyens afin de faire le nécessaire pour que leurs droits soient respectés dans un principe de "neutralité armée". Très rapidement, Théodore Pichon change de direction, impose la neutralité, puis agit pour empêcher les Français d'embrasser la cause des assiégés.

C'est également dans le contexte du siège qu'est né le périodique dont sont issus les textes en basque que nous présentons: Le Patriote Français. Journal commercial, littéraire et politique. Honneur et Patrie. Lors de son premier numéro', il justifie sa création. Ses auteurs veulent en faire le relais de l'ancien Messager (un journal publié par les résidents français mécontents lors de la signature du traité Mackau passé avec le dictateur argentin Rosas, traité qui lésa leurs intérêts commerciaux), ils ont une mission à accomplir: protéger les intérêts de leurs concitoyens résidant dans la république orientale, intérêts mis à mal par le traité de Mackau qui "venait réveiller encore une irritation presque assoupie et que le temps seul peut détruire." Le Patriote Français se révèle donc comme étant l'organe d'une population française en désaccord avec la politique menée par son gouvernement.

Le premier des quatre articles, paru deux fois ${ }^{2}$, invite à une réunion très importante et non armée les Français de la $5^{\text {emc }}$ section pour entendre le rapport de la commission et les mesures qu'elle a prises. Le lieu de rendez-vous est fixé "poste du jeu de Paume de Martin Cazenave, rue Saint Gabriel." L'avis émis en basque est immédiatement suivi de sa traduction.

Si le premier article est un appel de la commission formée autour du consul du roi, le second texte ${ }^{3}$ est signé par le colonel de la Légion française (Thiébaut). Une

\footnotetext{
${ }_{2}^{1}$ Le Patriote Français, mercredi $1^{\text {er }}$ et jeudi 02 février $1843, \mathrm{n}^{\circ} 1$, p. 2

2 Le Patriote Français, samedi 18 février $1843, n^{\circ} 14$, p. 3 ; dimanche 19 février $1843, n^{\circ} 15$, p. 1

${ }^{3}$ Le Patriote Français, lundi $1^{\text {er }}$ mai 1843 , n ${ }^{\circ} 71$, p. 2 ; vendredi 05 mai 1843 , n 73 , p. 2
} 
version française précède la version basque, conservant tout l'esprit du texte qui dans un même élan célèbre la fête du roi Louis-Philippe et condamne Théodore Pichon pour avoir abandonné seś compatriotes. Avec une verve très patriotique (du patriotisme français), le colonel Thiébaut appelle les Basques à être Français et à montrer que l'éloignement n'a point refroidi dans les cours l'amour de la patrie (dans la version française). Dans la version basque, il leur demande de se souvenir qu'ils sont français ("atchiquidezagun ohoresquy franses icena": conservons le souvenir du nom français) voire même basque-français ("hescualdun franses"). Ce discours est révélateur de l'attention portée aux Basques par les chefs militaires et d'une volonté de les associer au combat mené par les Français. Par contre, les promesses ne sont pas les mêmes pour tout le monde. De l'acquisition de 40.000 têtes de bétail annoncées en français, on passe à "hogoi eta borts mila" en basque, soit 15.000 animaux de moins saisis (quel sera alors le partage réalisé lors de la fin des combats, et comment sera-t-il effectué ?)

Dans les jours suivants paraît à quatre reprises, en version basque et française, le projet de la loi ${ }^{4}$ qui sera votée le 19 mai 1843 , loi qui récompensera les étrangers se battant pour la défense de Montevideo. Le dernier des quatre articles cités ne va paraître qu'une fois et uniquement en basques. Il s'intitule "Adaisquide eta herritar maitiac" (Compagnons et compatriotes aimés). Même s'il n'est pas signé, son contenu ne laisse aucun doute, il provient des Français de Montevideo. Ceux-ci s'interrogent dès le début sur l'action de leur consul face à la menace de Rosas, qualifié d'égorgeur ("odol ichurtçaleac"). La protection et les secours offerts par Monsieur Pichon ("Yaun Pichonec") sont jugés inexistants et le consul du roi est accusé d'employer comme il peut tous les moyens pour tromper la population. Théodore Pichon a également faussé le jugement de l'amiral français, amenant celui-ci à abandonner les siens et par ses mensonges le roi imagine que la prise d'armes des Français ne relève que d'un caprice. Face au danger, les rédacteurs n'envisagent qu'une seule solution, convaincre les lecteurs de s'enrôler à leurs côtés: "Moyen houra cen harmen hartcia eta harmatiac guria" (ce moyen est de prendre les armes et de faire notre armée). Dans leurs rangs, les rédacteurs peuvent tout de même compter sur le soutien d'un noble de haut rang ("Gure bocac adiarac tugu prince Joinvilly" : nous avons fait entendre notre voix au prince de Joinville) et sur la vigilance des Légionnaires.

Pour conclure, on peut dire que la parution de ces textes coïncide avec une période de course à l'armement dans la ville de Montevideo. Les Basques, tout comme les Français, qui ne prêtaient dans un premier temps aucune attention aux luttes américaines, s'y impliquèrent peu à peu, poussés à bout d'une part par les provocations et agressions de Rosas et attirés d'autre parts, par les promesses (dons de terres et exemptions d'impôts), du gouvernement assiégé. Le conflit, dans lequel ont pris part une Légion des volontaires français mais également un bataillon des Chasseurs basques, a débouché sur une défaite de leurs rivaux (Rosas et Oribe), défaite provoquée par l'intervention du général argentin d'origine basque Justo José de Urquiza.

\footnotetext{
${ }^{4}$ Le Patriote Français, lundi 08 mai $1843, \mathrm{n}^{\circ} 76$, p. 1 ; mercredi 10 mai $1843, \mathrm{n}^{\circ} 77$, p. 2 ; jeudi 11 mai $1843, \mathrm{n}^{\circ} 78$, p. 3 ; vendredi 12 mai $1843, \mathrm{n}^{\circ} 79$, p. 4

${ }^{5}$ Le Patriote Français, dimanche 17 septembre $1843, \mathrm{n}^{\circ} 185, \mathrm{p} .4$
} 


\section{Bibliographie :}

Capdehourat, Pélabère (auteurs présumés). Notes biographiques sur les chefs, sur les officiers, sur les meneurs et sur les plus distingués de la Légion Française de Montevideo, Miguelete, 1847. -1 vol., 14 pages.

Cooligan Maria Luisa, Arteaga Juan José. Historia del Uruguay desde los origenes hasta nuestros dias, Montevideo : Barreiro y Ramos, 1992.

De Santillan, Diego Abad. Historia argentina, Buenos Aires : Tipografica editora Argentina, 1981.--vol. 2, 569 pages ; vol. 3, 719 pages.

Piccirilli, Ricardo. Diccionario historico argentino (Tome V), Buenos Aires : Ediciones historicas argentinas, 1954, 920 pages.

Poucel, Benjamin. Les otages de Durazno. Souvenirs du Rio de la Plata pendant l'intervention Anglo-Française de 1845 à 1851, Paris, Achille Faure libraire éditeur, 1864, 86 pages.

Thiebaut, Jean-Chrisostôme. Le ministère du 15 août et la légion française, Montevideo : Imprimerie française, 1850. -1 vol., 33 pages. 


\section{Annexes :}

Premier texte

\section{MONTEVIDEO.}

\section{AVISA.}

Franges 5e sectionnecouter.

Frances appartenitcen direnacq 5en sectionniari, guimilatiacq dira oro reuniceco fattaric gabe, posta Martin Cascnaluren trinquetera, cailla san liabricllen, illabete honnen giten den igande 19 , hemeretei, egrorlice. beliartusten mesuren harcera, renuionnc hori arras imp rtanta la cesa ouklianen du audicnza armarice Gabe.

Aris uno l'rancuis de lu je seclion.

Les Francas appartenant á la 5e section, preste du jeu de Paume de Marrin Cayenave, rue Siant-(iorbricl, sont invilés á s'y lémuir tous. sans aucune cxerption, dimancbo prochain, lo lu comrant, á muili, pour y entendre: le rapport de diverses mesures urgentes prises par son comite. Cilte réunion est trés importante et aura licu sans armes.

Source: Le Patriote Français, samedi 18 février 1843, n 14, p. 3 ; dimanche 19 février $1843, \mathrm{n}^{\circ} 15, \mathrm{p} .1$ 


\section{Second texte}

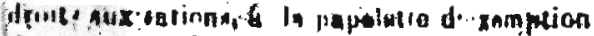

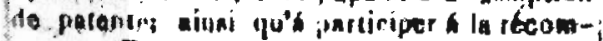
pethe offurie par le gonvornoment. Aurune

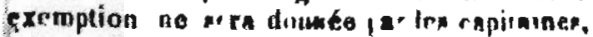

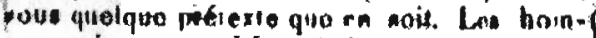

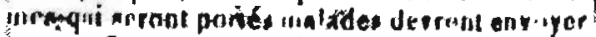

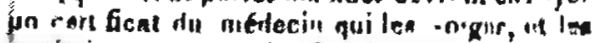

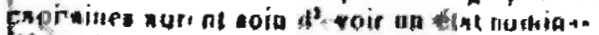

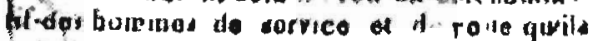
Tre reaur.
\end{abstract}

\section{PaOrer Da Lor.}

Art. 1. Le P. Fat est autnribé á procéder á lacquisition de 20 lienes carrées do terres Istonurables, den tioées á fonder des villes sur trois. poimsonu plús du littoral de la Ré. pirblfugue, au chioix du pouvirit exés eutif.

Azt. 2. Il est également altorisé á aquuerir 40,000 tétes de bótail. rt. 3 . Les terres el aninasux dint partent les articles précédens, - éront distribués a titre de récompunce entre tous les étrangers qui ont pris ori gui prendront les a rmes? ponr ta dafame do In caure de la Républiquie, et qui appartiendront aux leux légions de Volontairue Frangais et Italiuns.

\section{IECO ABOO PROTERA. Lehen Arfacta.}

Padore Erorucionesene motorisatain do

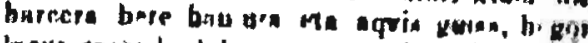

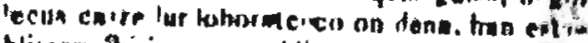

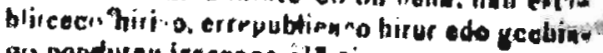
go ponduran irmannen alsinir.

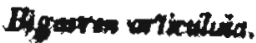

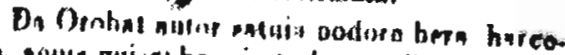

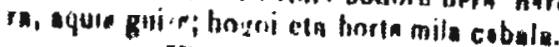

\section{Hirugerrea Anicukts.}

Erran larerae ar cabolue icanen dira bani.

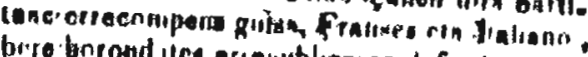

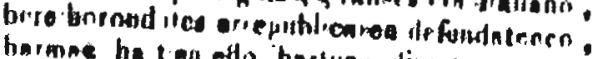

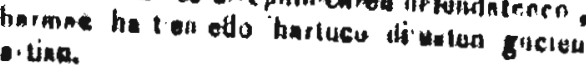

$$
\text { Largonmes Artiowion. }
$$

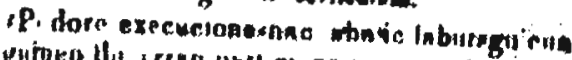

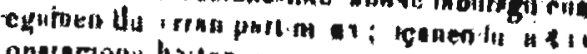

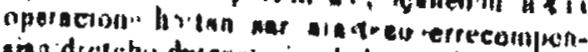

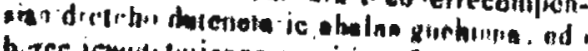

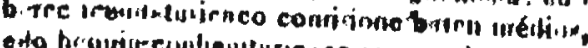

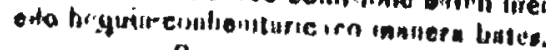

\section{Borlugarre Antindum.}

Pronnt ce projet h n ign dath comunis:

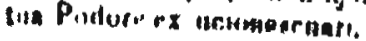

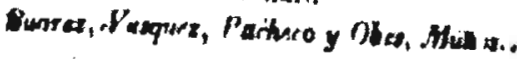

$$
\begin{aligned}
& \text { N UVILLES } 110 \text { COAN. }
\end{aligned}
$$

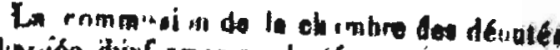

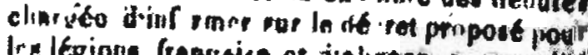

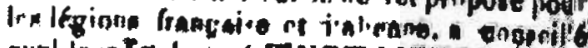

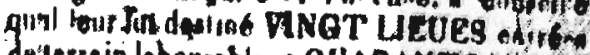

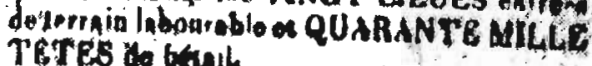

Source: Le Patriote Français, lundi 08 mai $1843, \mathrm{n}^{\circ} 76$, p. 1 ; mercredi 10 mai $1843, \mathrm{n}^{\circ} 77$, p. 2 ; jeudi 11 mai $1843, \mathrm{n}^{\circ} 78$, p. 3 ; vendredi 12 mai $1843, \mathrm{n}^{\circ} 79$, p. 4 
Troisième texte

CNMARADFS: + Su und nolitique imparọyante nona a frces le prendie lea atries pour u-tro délozo. gi lo Nécon do l'Amériquo a ofé nous menacer le wo, $t, n$ ón n cisonis pas colu dont nond tebrong nnjourhivi la féte.

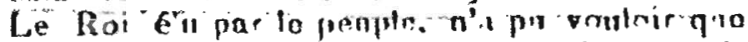

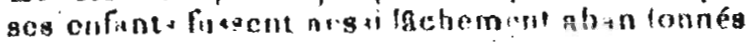
pur collit we no poli lied apola aus armea... cetuil lát est ang jatye!

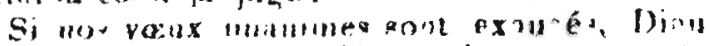

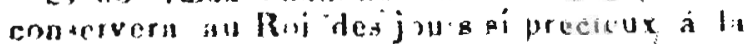

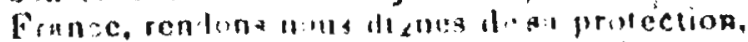

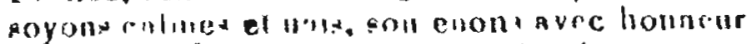

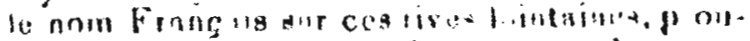
vons a nos ami-. a nos fores, th mème un.

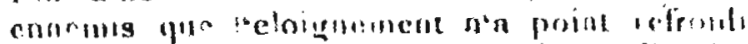

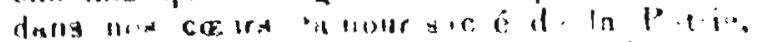

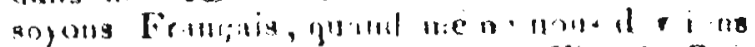

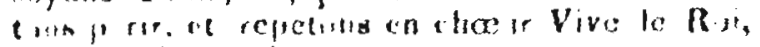
Vivela Frarce!

Le Colonel de la légion Fiançaise. TIIEISIU I,

\section{E.VE L.AGLS WISTIAC.}

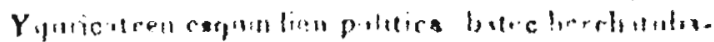

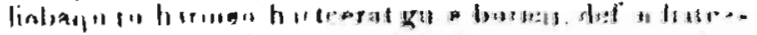

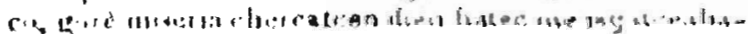

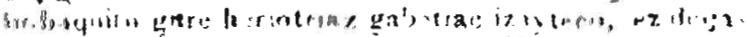

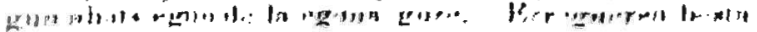

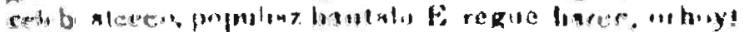

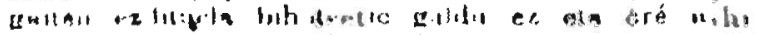

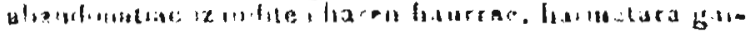

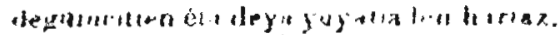

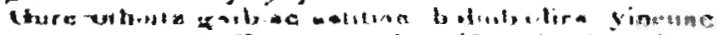

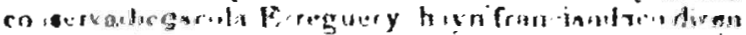

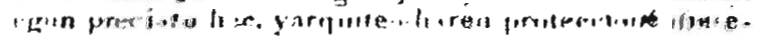

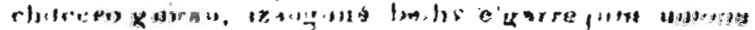

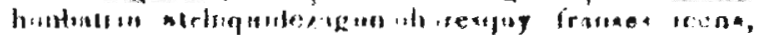

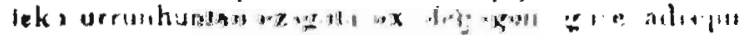

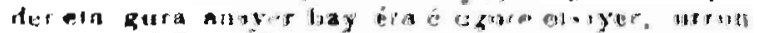

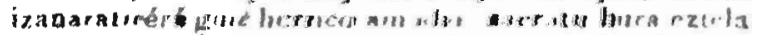

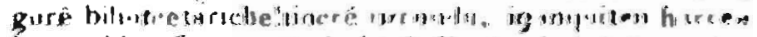

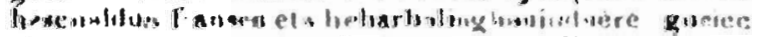

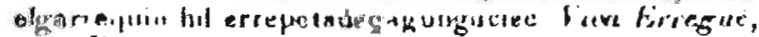
eica lirascia. 
Source: Le Patriote Français, lundi $1^{\text {tr }}$ mai 1843, n ${ }^{\circ} 71$, p. 2 ; vendredi 05 mai $1843, n^{\circ} 73$, p. 2

Quatrième texte

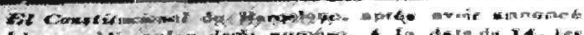

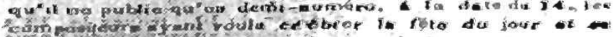

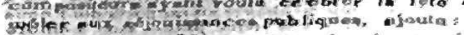

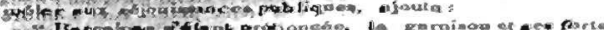

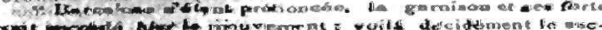

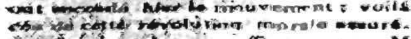

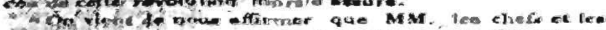

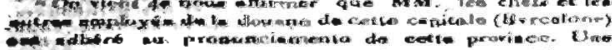

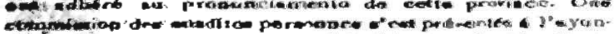

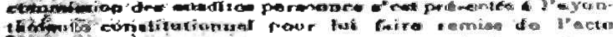

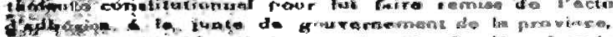

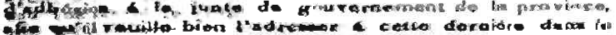

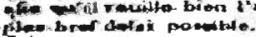

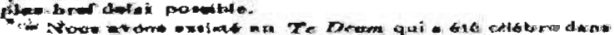

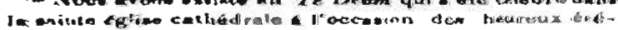
semeno dujour. Inouk serait dificile do decrire enco

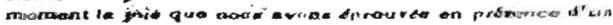

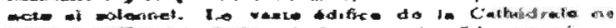

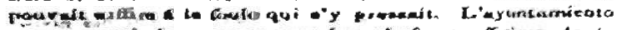

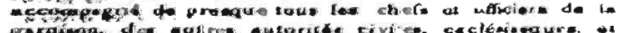

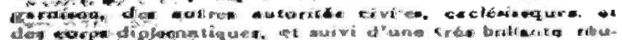

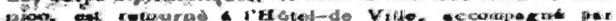

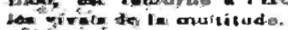

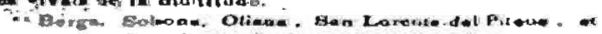

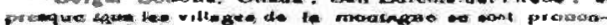

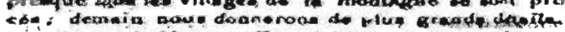

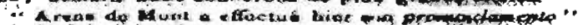

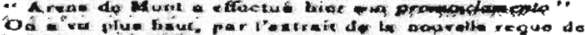

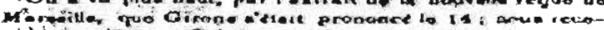

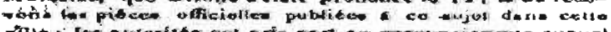

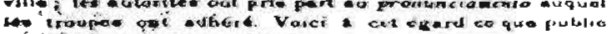

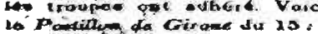

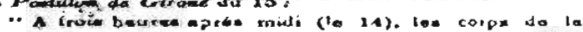

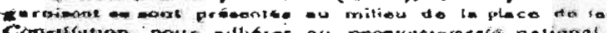

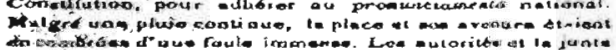

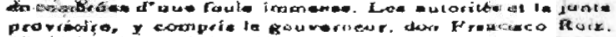

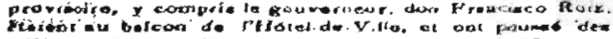

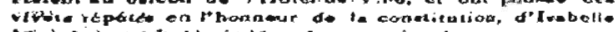

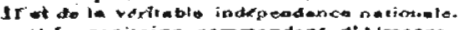

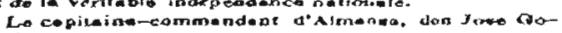

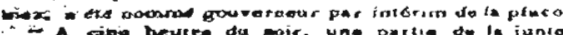

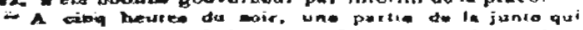

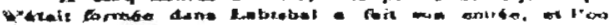

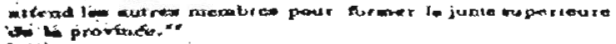

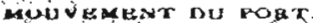

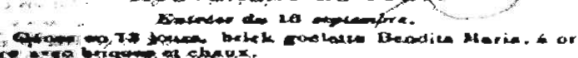

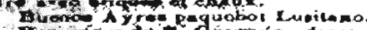

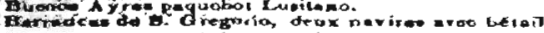

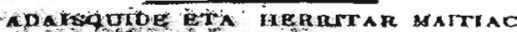

Noycótaoro tyrono faizo eta odul ichurtsalo hoe menergalcoo fuintionina ruinetcoco es

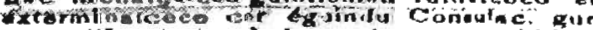

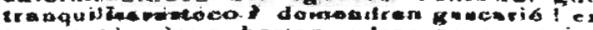

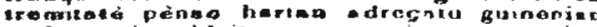

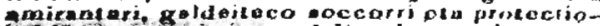

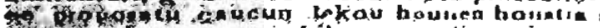

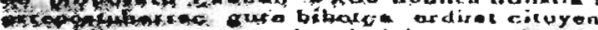

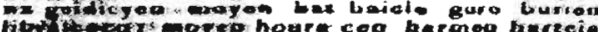

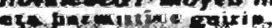

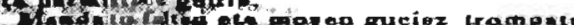

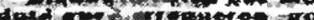

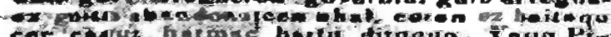

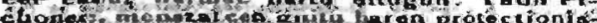

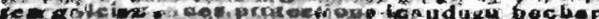
taino

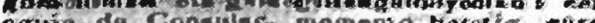

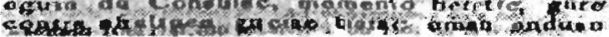
Feametcectidey

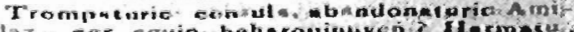
ralaz, cor eguin behnrauimuyenis Hexplast

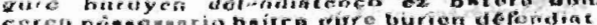
coro. coure haurron. gont eivation de farmilisen

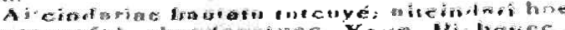

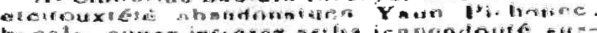

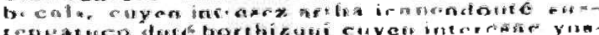

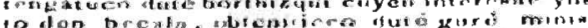

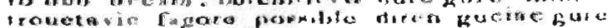

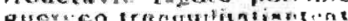

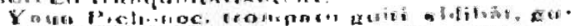

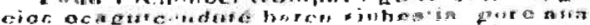

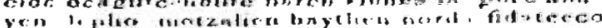

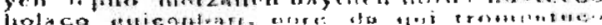

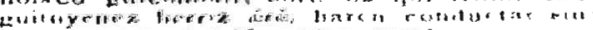

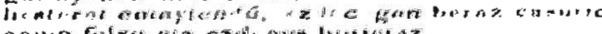

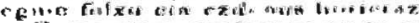

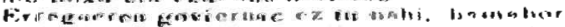

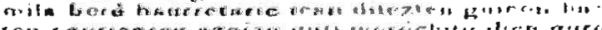

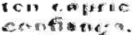

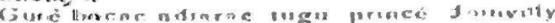

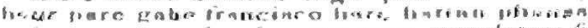

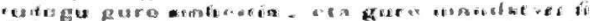

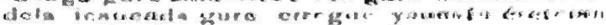

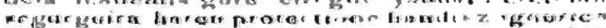

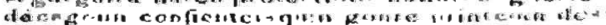

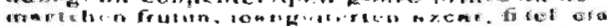

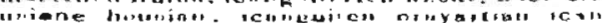

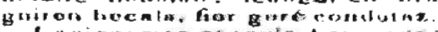

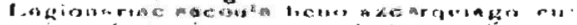

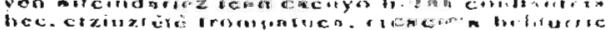

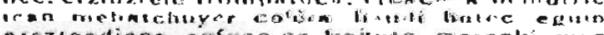

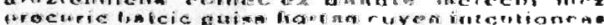

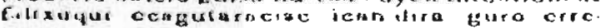
gerte?

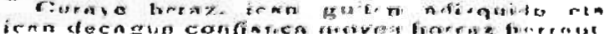

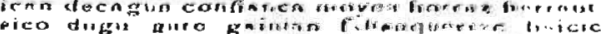

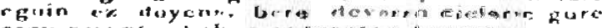

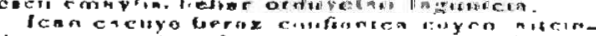

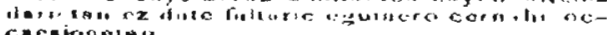

\section{AVIS IDIVIRS}

\section{A VI 1 .}

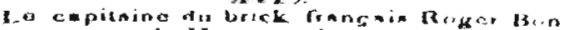

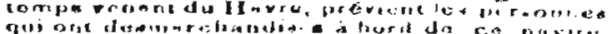

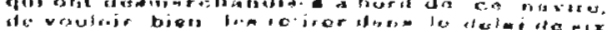

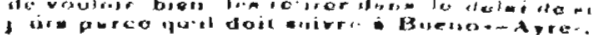

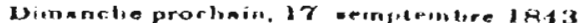

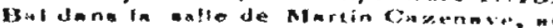

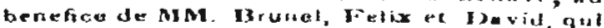
no stegligerome rion pous yue low ambceure mient anciurmis.

Loreliebro area caropuea comme pur te

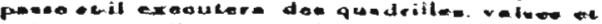

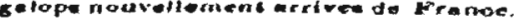

Le bel nur. lien cou les dienariches ef fourt to folo depuin 2 houres do lepres anidi JuFqu" lowitheuren du eqir.

Prix dentrab $1 \mathrm{a}$ veintales

\section{I. directeur it in anilo}

BIt U N I I L

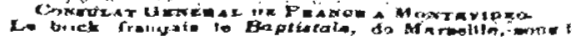


Source : Le Patriote Français, dimanche 17 septembre 1843, nº 185 , p. 4

\title{
Transcription du premier texte
}

\author{
AVISA \\ FRANCES $5^{5}$ SECTIONNECOUER.
}

Frances appartenitcen direnacq 5en sectionnari, guimitatiacq dira oro reuniceco fattarie gabe, posta Martin Casenabaren trinquetera, cailla San Gabriellen, illabete honnen giten den igande 19, hemeretei, egordico, behartusten mesuren harcera, reunionne hori arras importanta da esa oukhanen du audienza armaricq gage.

AVIS AUX FRANÇAIS DE LA $5^{\mathrm{E}}$ SECTION.

Les Français appartenant á la $5^{\mathrm{e}}$ section, poste du jeu de Paume de Martin Cazenave, rue Saint Gabriel, sont invités á s'y réunir tous, sans aucune exception, dimanche prochain, $19 \mathrm{du}$ courant, á midi, pour y entendre le rapport de diverses mesures urgentes prises par son comité. Cette réunion est trés importante et aura lieu sans armes.

\section{Transcription du second texte}

\section{PROJET DE LOI.}

Art. 1. Le P. E. est autorisé à procéder à l'acquisition de 20 lieues carrées de terres labourables, destinées à fonder des villes sur trois points ou plus du littoral de la République, au choix du pouvoir exécutif.

Art. 2. Il est également autorisé à acquérir 40.000 têtes de bétail.

Art. 3. Les terres et animaux dont parlent les articles précédents, seront distribués à titre de récompense entre tous les étrangers qui ont pris ou qui prendront les armes pour la défense de la cause de la République, et qui appartiendront aux deux légions de Volontaires Français et Italiens.

Art. 4. Le pouvoir exécutif procédera dans les plus brefs délais á la répartition susmentionnée, il prendra soin de donner dans cette opération la plus grande intervention possible á ceux qui ont droit aux récompenses, soit au moyen d'une commission par eux nommée, soit d'une manière convenue avec eux.

Art. 5. Que le présent projet soit communiqué au P. E.

Suarez, Vasquez, Pacheco y Obes, Muñoz

LEGUESCO PROJETA.

Lehen Articulia.

Podore Execucionescua autorisatua da harcera bere hautura eta aquis guisa hegoi lecua carre lur loboratceco on dena, han establitceco hiriac, errepublieco hirur edo guchiago pondutan ixasoaco aldetic.

\section{Bigarren articuluia} cabala.

Da Orobat autorisatuia podore bera harcera, aquis guisa ; hogoi eta borts mila 


\section{Hirugarren Articuluia}

Erran lurrac eta cabalac içanen dira bartituac errecompens guisa, Franses eta Italiano, bere borondates errepublicaren defendatceco, harmaz hat en edo hartuco diusten gucien artian.

\section{Laurgarren articuluia}

Podore execucionescuac ahatic labureguiena eguimen du erran partimean : içanen du [???] operacione hartan sar sar arasteco errecompensian dretcho dutenetaric ahalas guehiena, edo berec icedatutenco condicione baten médios, edo heguin conbertaricaco manera bates.

\section{Bortsgarren Articuluia}

Present co projet han içan daila comunicatua Podore escucionescuari.

Suarez, Vasquez, Pacheco Y Obes, Muñoz.

\section{Transcription du troisième texte}

CAMARADES :

- Si une politique imprévoyante nous a forcé de prendre les armes pour notre défense, si le Néron de l'Amérique a osé nous menacer de mort, n'en accusons pas celui dont nous célébrons aujourd'hui la fête. Le Roi élu par le peuple, n'a pu vouloir que ses enfants fussent aussi lâchement abandonnés par celui même qui les appela aux armes...celui lá est déjà jugé !

Si nos vœux unanimes sont exaucés, Dieu conservera au Roi des jours si précieux á la France, rendons nous dignes de sa protection, soyons calmes et unis, soutenons avec honneur le nom Français sur ces rives lointaines, prouvons à nos amis, à nos frères, et même nos ennemis que l'éloignement n'a point refroidi dans nos coeurs l'amour sacré de la Patrie, soyons Français, quand même nous devrions tous périr, et répétons en chœur Vive le Roi, Vive la France !

\section{Le Colonel de la Légion Française.}

THIEBAUT

\section{ENE LAGUN MAYTIAC.}

Yquricatcen esquindien politica batec berchatubalioquta harmen hartcerat gure burien, defendatceco, guré miseria chercatcen dien batec menaçatceabalinbaquitu gure heriotciaz gabetiac izayteco, ez dezagun ahats egun dela eguna gure. Erregueren besta celebratceco, populiaz bautalu Erregue harec, orhoyt gaiten ez dituela bihotcetic galdu ez eta éré nahi abandonatiac izanditen haren haurrac, harmetara galdeguincitien éta deya yuyatia den hartaz.

Gure orhoitz garbiac estitian balinbadira yincuac conservadieçacola Erreguery hayn franciandaco diren egun preciatu hec, yarquite haren protectioné merechitceco guiran, izanguité bachy elgarrequin unione honbaitan atchiquidezagun ohoresquy frances icena, leku urrunhuntan ezagatu deçagun gure adisquider eta gura anayer bay éta ére gure etsayer, urrun izanaraticéré guré herrico amodio sacratu hura eztela guré bihotcetaricbehineré urrundu, içanquiten harren hescualdun franses eta beharbalinbadinduéré guciec elgariequin hil erepetadeçagunguciec Viva Erregué, viva Francia. 


\section{Transcription du quatrième texte}

\section{Adaisquide eta herritar maitiac}

Noycetaere tyrano falzo eta odol ichurtçale hac menatçalcen guintienian ruinatceco et exterminatceco cer eguindu Consulac, gur tranquilisarasteco ? demendren gaucarié ! extremitate penae hartan adreçatu guinenian amirantari, galdeiteco soccorri eta protectione proposatu çaucun lekou hounen houstia : errepostuharrec gure bihotça erdirat cituyen ez guidieyen moyen bat baicie gure burien libratceco : moyen houra cen harmen hartcia eta harmatiac guiria.

Mandatu faltsu eta moyen guciez trompatu dute gure erregueren governia : gure erregue ez guitu abandonatcen ahal, ceren ez baita cer casuz harmac hartu ditugun. Yaun Pichonec, menazatcen guitu haren protectioniaren galciaz. Cer protectione $i$ udugu behar guindreman eta galdeguitenguinuyonian! cer eguin du Consulac, memento beretic, gure contra ahalzuen guciac berac eman onduan hermatceco ideya.

Trompaturic consula, abandonaturic Amiralaz, cer eguin beharquinuyen? Harmatu gure buruyen defendiatceco ez batere dolu ceren nessessario baitce gure burien defendiatceco, goure haurren, goure emasten eta familien.

Aitcindariac hautatu tutcuyé : aitcindari hoc etcitouxtété abandonatuco Yaun Pichonec becala, cuyen intresez artha icanendouté sustengatuco duté bothizqui cuyen interesec yusto don becala, obtenitieco dutè gurè ministrouetaric fagore possible diren guciac gure guereco tranquilitatiantcat.

Yaun Pichonec, trompatu guiti aldibat, guciec ecagutcenduté haren sinhestia gure anayen lepho metzalien baythen norda fidatceco holaco guiconbati, norc da qui trompatuco guituyenez berriz èrè, haren conductac sinhesterat emaytendu, ezdecagun beraz casume eguin falxu eta ezdous horietaz.

Erregueren goviernac ez tu nahi, hamabortmila berè haurretaric ican ditezten guicon baten capricaren azpian guc merèchitu dien gure confiança.

Gure bocac adiarac tugu princè Joinvilly haur pare gabe tranciaco hau, hartan phausa tudugu gure sinhestia, eta gure mandatari fidela içanenda gure erregue yaunaia eretcian segurguira haren protectione handiz igourica dècagoun confientciaquin goure printcean desmartchen frutun, icanguiterten azcar, fidel eta unione hounian, icanguiren orayartian ican guiren becala, fier guré condutaz.

Legionariac secoula beno azcarquiago cuyen aitcindariez ican cacuyo hetan confiantcia hec, etziustètè trompatuca, etcaciela beldurric ican mehatchuyer colèra handi batec eguin araztendiena coinec ez baitute merechi mezprecuric baicic guisa hortan cuyen intentioneas faltxuqui ecagutaraciac ican dira gure erregueri.

Curaye beraz, ican guiten adisquide eta ican decagun confianca moyen horraz berrautsico dugu gure gainian faltsuqueriac baicic eguin ez duyena, bere devarra cielaric gure escu emaytia, behar orduyetan laguntcia.

Ican escuye beraz confiantca cuyen aitcindarietan ez dute falturic eguinero cernahi occaesionetan. 